\title{
Transfusion-Related Acute Lung Injury: What the Allied Health Professional Needs to Know about this Life -Threatening Hazard of Blood Transfusion
}

\author{
Kevin P. Collins \\ Texas State University, kc35@txstate.edu \\ Thomas L. Patterson \\ tp20@txstate.edu
}

Follow this and additional works at: https://nsuworks.nova.edu/ijahsp

Part of the Medicine and Health Sciences Commons

\begin{abstract}
Recommended Citation
Collins KP, Patterson TL. Transfusion-Related Acute Lung Injury: What the Allied Health Professional Needs to Know about this Life -Threatening Hazard of Blood Transfusion. The Internet Journal of Allied Health Sciences and Practice. 2014 Apr 01;12(2), Article 4.
\end{abstract}

This Manuscript is brought to you for free and open access by the College of Health Care Sciences at NSUWorks. It has been accepted for inclusion in Internet Journal of Allied Health Sciences and Practice by an authorized editor of NSUWorks. For more information, please contact nsuworks@nova.edu. 


\title{
Transfusion-Related Acute Lung Injury: What the Allied Health Professional Needs to Know about this Life -Threatening Hazard of Blood Transfusion
}

\begin{abstract}
Transfusion-Related Acute Lung Injury (TRALI) is a rare, life-threatening hazard of blood transfusion. In the intensive care unit, $37 \%$ to $44 \%$ of admitted patients are transfused with at least one blood component. The opportunity for health professionals and students from nursing, respiratory care, and clinical laboratory science to interact directly with patients receiving a blood transfusion is likely to present itself on a daily basis. It is imperative for those caring for critically ill patients to be fully aware of the clinical signs of TRALI and have knowledge in the prevention and treatment of this clinical syndrome. Common clinical signs of TRALI include cough, fever, and dyspnea. Treatment has been mostly supportive and aimed at reversing acute respiratory distress through oxygen therapy or mechanical ventilation. In 2004, a consensus panel and the U.S. National Heart, Lung, and Blood Institute (NHLBI) working group characterized TRALI as newonset acute lung injury (ALI) occurring within six hours of transfusion in patients without preexisting ALI. Although the condition is uncommon, knowledge of this clinical syndrome may help identify those patients at risk and may prevent lung injury and possible death due to this rarely encountered hazard of blood transfusion.
\end{abstract}

\section{Author Bio(s)}

- Kevin Collins, MS, RRT, RPFT, AE-C, is an Assistant Professor in the Department of Respiratory Care at Texas State University, San Marcos.

- Thomas Patterson, MS, MT (ASCP), is an Assistant Professor in the Clinical Laboratory Sciences Program as Texas State University, San Marcos. 


\title{
IJAHSP \\ The Internet Joưnal of Allied Health Sciences and Practice \\ Dedicated to allied health professional practice and education
}

Vol. 12 No. 2 ISSN 1540-580X

\section{Transfusion-Related Acute Lung Injury: What the Allied Health Professional Needs to Know about this Life -Threatening Hazard of Blood Transfusion}

\author{
Kevin P. Collins, MS, RRT, RPFT, AE-C \\ Thomas L. Patterson, MS, MT (ASCP)2
}

1. Assistant Professor, Department of Respiratory Care, Texas State University, San Marcos

2. Assistant Professor, Clinical Laboratory Sciences Program, Texas State University, San Marcos

United States

\begin{abstract}
Transfusion-Related Acute Lung Injury (TRALI) is a rare, life-threatening hazard of blood transfusion. In the intensive care unit, $37 \%$ to $44 \%$ of admitted patients are transfused with at least one blood component. The opportunity for health professionals and students from nursing, respiratory care, and clinical laboratory science to interact directly with patients receiving a blood transfusion is likely to present itself on a daily basis. It is imperative for those caring for critically ill patients to be fully aware of the clinical signs of TRALI and have knowledge in the prevention and treatment of this clinical syndrome. Common clinical signs of TRALI include cough, fever, and dyspnea. Treatment has been mostly supportive and aimed at reversing acute respiratory distress through oxygen therapy or mechanical ventilation. In 2004, a consensus panel and the U.S. National Heart, Lung, and Blood Institute (NHLBI) working group characterized TRALI as new-onset acute lung injury (ALI) occurring within six hours of transfusion in patients without preexisting ALI. Although the condition is uncommon, knowledge of this clinical syndrome may help identify those patients at risk and may prevent lung injury and possible death due to this rarely encountered hazard of blood transfusion.
\end{abstract}

\section{INTRODUCTION}

In the acute care hospital, blood transfusions are utilized by physicians on a daily basis. ${ }^{1}$ Transfusion of whole blood or one of the components of blood is usually completed in an uneventful manner; however, some recipients may experience complications that range from mild to life threatening, including severe hypoxemia, bilateral pulmonary edema, and allergic reactions. ${ }^{2}$ For health professionals and students caring for critically ill patients, it is important to be fully aware of the hazards of blood transfusion. In the intensive care unit (ICU), 37 to $44 \%$ of admitted patients are transfused with at least one blood component. ${ }^{3}$ The opportunity to interact directly with patients receiving a blood transfusion in the ICU is likely to present itself to health professionals and students from nursing, respiratory care, and clinical laboratory science. This article will examine transfusionrelated acute lung injury (TRALI); a life-threatening complication of blood transfusion. ${ }^{4}$ Included in this discussion will be the incidence, definition, pathophysiology, prevention, and treatment of TRALI.

\section{INCIDENCE}

TRALI represents a leading cause of transfusion-related deaths. $.6,6$ In the United States, between 2003 and 2005, there were 550 reported cases of TRALI, but it is suspected that there may be many more cases that were not reported. 7,8 Incidence is difficult to estimate because there are no published large clinical trials of TRALI. However, estimates range from 1 in 300 to 1 in 5000 transfusions. ${ }^{9}$ The incidence of TRALI is the same for males and females, and it has been known to occur in patients of all ages.10,11 A correct diagnosis of TRALI can be difficult because of a variety of pulmonary complications and other types of transfusion reactions that may easily interfere with the diagnosis. An incidence of acute lung injury (ALI) may not readily be associated with the transfusion of plasma or other blood products the patient may be receiving as the etiology of TRALI is complex and is not always clear-cut. 


\section{DEFINITION}

TRALI is defined as a clinical syndrome, not a disease arising from a single cause. ${ }^{12}$ The difficulty in diagnosing TRALI is a result of the lack of a "gold standard" diagnostic tool. ${ }^{1}$ Therefore, a diagnosis relies on the assessment of clinical symptoms (e.g., dyspnea, cough, fever) and the exclusion of other transfusion reactions. ${ }^{13}$ In diagnosing TRALI, the healthcare practitioner should exclude the most common reason for pulmonary edema and subsequent respiratory distress in a patient receiving a blood transfusion, transfusion-associated circulatory overload (TACO). TACO is important to rule out because it can mimic TRALI as both are associated with pulmonary edema. ${ }^{2}$ Another reason TRALI may be difficult to diagnose is the complex picture presented by overlying disease processes (e.g., pneumonia, severe sepsis, shock) that may be occurring at the same time..$^{12} \mathrm{~A}$ consensus panel and the U.S. National Heart, Lung, and Blood Institute (NHLBI) working group convened in 2004 to develop a clinical definition for this syndrome. The definition is based on clinical and radiological parameters developed by the consensus panel and are presented in table 1.1

\begin{tabular}{|l|} 
Table 1: Definition of TRALI \\
\hline Suspected TRALI \\
\hline Acute onset within 6 hours of blood transfusion \\
\hline Bilateral infiltrates changes on chest radiograph \\
\hline PaO2/FIO2 $\leq 300 \mathrm{mmHg}$, or worsening of $\mathrm{P}$ to $\mathrm{F}$ ratio \\
\hline $\begin{array}{l}\text { No sign of hydrostatic pulmonary edema (PAOP } \leq 18 \mathrm{mmHg} \text { or central venous } \\
\text { pressure } \leq 15 \mathrm{mmHg} \text { ) }\end{array}$ \\
\hline No other risk factor for acute lung injury \\
\hline Possible TRALI \\
\hline Same as for suspected TRALI, but another risk factor present for ALI \\
\hline Delayed TRALI \\
\hline Same as for (possible) TRALI and onset within 6-72 hours of blood transfusion \\
\hline
\end{tabular}

$A L I=$ acute lung injury; $F I O 2$ = fraction of inspired oxygen; $P a O 2$ = arterial oxygen, $P A O P=$ pulmonary artery occlusion pressure; TRALI = transfusion-related acute lung injury.

The consensus panel characterized this syndrome as new-onset acute lung injury occurring within six hours of transfusion in patients without preexisting ALI. ${ }^{1} \mathrm{ALI}$ is defined by the North American European Consensus as new-onset hypoxemia with bilateral pulmonary infiltrates occurring on the chest radiograph in the absence of volume overload and a blood oxygen saturation of $90 \%$ or less on room air. ${ }^{14}$ Due to the lack of specific diagnostic markers, the consensus panel recommends utilizing the definition in Table 1 for clinical practice and research. ${ }^{15}$

\section{PATHOPHYSIOLOGY}

A diagnosis of TRALI is strongly supported after finding anti-HLA antibodies (antibodies to human leukocyte antigens) or other anti-leukocyte antibodies in the donor's plasma. ${ }^{16}$ It is currently believed that one cause of TRALI is the transfer of anti-human leukocyte antibodies from the donor to the recipient that may cause primed leukocytes in the recipient's lungs to de-granulate, setting up an acute inflammatory response in the lung parenchyma. The resulting capillary leakage is likely the inflammatory process that causes the associated pulmonary edema. ${ }^{17}$ This hypothesis is supported by look-back studies that show that most cases of TRALI are associated with transfusions of plasma or other components that contain plasma. ${ }^{9}$ To further support the donor antibody hypothesis, it has been noted that plasma products from female multiparous donors, or women that have had two or more pregnancies, pose a greater risk for TRALI than products from male donors. ${ }^{18}$ This suggests that female multiparous donors that have been repeatedly exposed to fetal HLA antigens may generate a wide variety of HLA antibodies or antineutrophil antibodies. Theoretically, plasma from male donors would contain very little, if any, of these types of antibodies unless the donor had previously received multiple blood transfusions. In 2003, the United Kingdom blood system recommended eliminating from inventory the fresh frozen plasma of female donors. ${ }^{19}$ Donor centers in the United States soon followed, preferring male only donors for the production of plasma products. ${ }^{20}$

There is also evidence to suggest that TRALI may have another cause. In some cases of TRALI, there have been no antibodies associated with this condition. Investigation in this area has created an interesting hypothesis called the "two hit" theory. In this model, the first "hit" is a predisposing factor that causes circulating leukocytes to adhere to the capillary endothelium in the alveoli. This insult can be caused by pneumonia, surgery, trauma, near drowning, or massive transfusion. The pulmonary 
leukocytes are then stimulated by the second "hit" of priming molecules causing the sequestered leukocytes to release oxidases and proteases with resulting damage to the capillary endothelium. It is this immunological damage that causes leakage of fluid into the air spaces. ${ }^{5}$

These priming molecules called the "second hit" are contained in the transfusion. Priming lipids are biological response modifiers that can bind to receptors on sequestered leukocytes and initiate degranulation. It has been demonstrated that soluble CD40 molecules may also be responsible for the second hit that initiates leukocyte activation. Components containing red blood cells shed larger amounts of these types of molecules into the plasma. ${ }^{21}$ These molecules are believed to be breakdown products released from platelets and red blood cell membranes in the component bag.

Biological response modifiers tend to accumulate over time in stored blood components. The length of storage time is directly related to the amount of these molecules that are present in the plasma fraction of the component. One study has demonstrated that red cells stored for as little as 15 days under recommended storage conditions could produce enough priming molecules to activate neutrophils, while red cells stored for 35 days showed the greatest propensity for stimulating neutrophils. ${ }^{22}$ Animal studies have also supported the two hit hypothesis. Lungs of rats were pretreated with lipopolysaccharide endotoxin to adhere neutrophils to the capillary endothelium. Plasma from fresh blood and from blood stored at 42 days (the normal outdate period for red blood cells) was then perfused into the pretreated lungs and the rats were monitored for acute lung injury. Rat lungs perfused with plasma from a freshly drawn blood unit did not demonstrate ALI while rat lungs perfused with plasma extracted from 42 day-old red blood cells showed significant pulmonary edema. ${ }^{23}$

\section{TREATMENT}

The treatment of TRALI is mostly supportive and aimed at reversing acute respiratory distress through oxygen therapy, noninvasive positive pressure ventilation by mask, (e.g., continuous positive airways pressure), or mechanical ventilation. In 70 to $90 \%$ of cases, patients require endotracheal intubation with subsequent mechanical ventilation. TRALI is categorized as a form of acute lung injury thereby utilizing a lung-protective strategy with low tidal volumes (6 to $8 \mathrm{ml} / \mathrm{kg}$ predicted body weight) is recommended. ${ }^{1}$ Mechanical ventilation (MV) can be a lifesaving intervention; however, one source reveals that MV may induce or aggravate lung injury known as ventilator induced lung injury $(\mathrm{VILI}) \cdot{ }^{23} \mathrm{It}$ has been reported in one study that up to $33 \%$ of mechanically ventilated critically ill patients developed lung injury within 48-hours of transfusion. ${ }^{4}, 24$ Vlaar et al reported in an animal study that MV may prime pulmonary neutrophils, putting the lungs at risk for a TRALI reaction. ${ }^{4}$

In a case report by Huang et al, the authors reported a patient with severe TRALI who, failing conventional mechanical ventilation, was successfully transferred to High-Frequency Oscillatory Ventilation (HFOV) for rescue from refractory hypoxemia. ${ }^{25}$ Huang et al showed HFOV was effective in correcting refractory hypoxemia in two days and the patient was transferred back to conventional mechanical ventilation with ventilator liberation in two weeks. ${ }^{25}$ The authors suggest HFOV may be considered as a rescue therapy for severe TRALI patients not responding to conventional mechanical ventilation. Other strategies that have proven to enhance improvements in oxygenation in patients with TRALI include prone position, nitric oxide inhalation, and extracorporeal membrane oxygenation. ${ }^{25}$ Pharmacologic therapy used to treat TRALI includes systemic corticosteroids, diuretics, and pressors. Use of systemic corticosteroids to reduce inflammation in the lungs does not have supporting evidence to prove benefits or effectiveness. ${ }^{26}$ The inhaled corticosteroid budesonide is being studied as a preventative strategy in a multicenter evaluation as a nebulized solution used for patients at high risk for ALI. ${ }^{27}$ Diuretics may show promise in maintaining a restrictive fluid strategy that is beneficial in ALI; however, another source states the use of diuretics to treat TRALI may be harmful, resulting in decreased cardiac output and may contribute to hypotension. ${ }^{1,2}$ For those patients with prolonged hypotension, the use of pressor agents may be required to maintain blood pressure. ${ }^{2}$

\section{PREVENTION}

Patients with previous lung injury may be predisposed to develop TRALI if they receive a transfusion of plasma containing products, whole blood, or even packed red blood cells. ${ }^{9}$ It would be prudent to be conservative in the transfusion of blood or plasma to these patients. If transfusion is absolutely necessary, plasma from male donors is always the first choice, and in fact, most donor centers do not process plasma from multiparous female donors. ${ }^{20}$ If red cells are needed to boost oxygen carrying capacity, fresh red blood cells should be transfused, as it has been shown that fresher units contain smaller amounts of neutrophil priming lipids. ${ }^{22}$ Fresh red blood cells should be requested from the blood bank, so the laboratory must be made aware of the risk to the patient.

It would seem that one central issue in the prevention and treatment of TRALI is medical education. Recognition and treatment of TRALI crosses several disciplines: nursing, respiratory care, and the clinical laboratory. Nursing personnel are the front line caregivers and must be able to quickly identify the signs and symptoms of ALI associated with transfusion. The respiratory care practitioner must be made aware of those patients receiving a blood transfusion in order to be prepared to administer the

(C) The Internet Journal of Allied Health Sciences and Practice, 2014 
appropriate therapy including monitoring the oxygenation and ventilation status of the patient. The blood bank in the clinical laboratory should be alerted to utilize the freshest blood to be cross-matched for patients with high risk factors for TRALI. It is imperative that every department involved understands the signs, symptoms, and pathology associated with TRALI and effectively communicates with each other. The standard of care would include informing the attending physician of any signs or symptoms of ALI associated with transfusion.

\section{CONCLUSION}

As patient advocates, health professionals should have knowledge of the clinical signs of TRALI, be able to recognize the condition, and must report any unusual reactions for patients receiving a blood transfusion to the attending physician. In an effort to increase awareness of TRALI, the educational curricula for nursing, respiratory care, and clinical laboratory science should include an instructional module on the etiology, prevention, and treatment of this clinical syndrome. Although the condition is uncommon, knowledge of TRALI may help identify those patients at risk and may prevent lung injury and possible death as a result of this rarely encountered hazard of blood transfusion.

\section{REFERENCES}

1. Vlaar AP, Juffermans NP. Transfusion-related acute lung injury: a clinical review. Lancet. 2013:382(9896):984-94. [PMID 23642914]

2. Kopko PM. Transfusion-related acute lung injury. J Infus Nurs. 2010;33(1):32-7. [PMID 20075682]

3. Benson AB, Austin GL, Berg M, McFann KK, Thomas S, Ramirez G, et al. Transfusion-related acute lung injury in ICU patients admitted with gastrointestinal bleeding. Intensive Care Med. 2010;36(10):1710-7. [PMID 20658125]

4. Vlaar AP, Wolthuis EK, Hofstra JJ, Roelofs JJ, Boon L, Schultz MJ, et al. Mechanical ventilation aggravates transfusion-related acute lung injury induced by MHC-1 class antibodies. Intensive Care Med. 2010;36(5):879-87. [PMID 20221752]

5. Silliman CC, Ambruso DR, Boshkov LK. Transfusion related acute lung injury. Blood. 2005;105(6):2266-73. [PMID 15572582]

6. Vlaar AP, Wortel K, Binnekade JM, van Oers MH, Beckers E, Gajic O, Schultz MJ, Juffermans NP. The practice of reporting transfusion related acute lung injury. Transfusion. 2010;50(2):443-51. [PMID 19804567]

7. Eder AF, Herron R, Strupp A, Dy B, Notari EP, Chambers LA, et al. Transfusion-related acute lung injury surveillance (2003-2005) and the potential impact of the selective use of plasma from male donors in the American Red Cross. Transfusion. 2007;47(4):599-607. [PMID 17381617]

8. Wallis JP. Transfusion-related acute lung injury: underdiagnosed and under reported. Br J Anaesth. 2003;90(5):573-6. [PMID 12697582]

9. Popovsky MA, Moore SB. Diagnostic and pathogenic considerations in transfusion-related acute lung injury. Transfusion. 1985;25(6):573-7. [PMID 4071603]

10. Popovsky MA, Haley NR. Further characterization of transfusion-related acute lung injury: demographics, clinical and laboratory features, and morbidity. Immunohematology. 2000;16(4):157-9. [PMID 15373608]

11. Popovsky MA, Chaplin HC Jr, Moore SB. Transfusion-related acute lung injury: a neglected, serious complication of hemotherapy. Transfusion. 1992;32(6):589-92. [PMID 1502715]

12. Kleinman $S$, Gajic $O$, Nunes E. Promoting recognition and prevention of transfusion-related acute lung injury. Critical Care Nurse. 2007;27(4):49-53. [PMID 17671245]

13. Cherry T, Steciuk M, Reddy VV, Marques MB. Transfusion-related acute lung injury: past, present, and future. Am J Clin Pathol. 2008;129(2):287-97. [PMID 18208810]

14. Maker RS, Powers A, Stowell CP. Reducing transfusion-related acute lung injury risk: evidence for and approaches to transfusion-related acute lung injury mitigation. Transfusion Medicine Reviews. 2012;26(4):305-20. [PMID 22520553]

15. Rana R, Fernandez-Perez ER, Khan SA, Rana S, Winters JL, Lesnick TG, et al. Transfusion-related acute lung injury and pulmonary edema in critically ill patients: a retrospective study. Transfusion. 2006;46(9):1478-83. [PMID 16965572]

16. Kleinman $\mathrm{S}$, Caulfield $\mathrm{T}$, Chan $\mathrm{P}$, et al.. Towards an understanding of transfusion-related acute lung injury: a statement of a consensus panel. Transfusion. 2004;44(12):1774-89. [PMID 15584994]

17. Popovsky MA, Abel MD, Moore SB. Transfusion-related acute lung injury associated with passive transfer of antileukocyte antibodies. Am Rev Respir Dis.1983;128(1):185-9. [PMID 6603182]

18. Palfi M, Berg S, Ernerudh J, Berlin G. A randomized controlled trail of transfusion-related acute lung injury: is plasma from multiparous blood donors dangerous? Transfusion. 2001;41(3):317-22. [PMID 11274583]

19. England bans FFP from female donors to prevent TRALI. ABC Newslett. 2003;(44):1-5. 
20. Strong DM, Shoos Lipton K, Transfusion-related acute lung injury. AABB Bulletin \#06-07 (November 3, 2006) http://www.aabb.org/resources/publications/bulletins/Pages/ab06-07.aspx Accessed November 14, 2013.

21. Kahn SY, Kelher MR, Heal JM, Blumberg N, Boshkov LK, Phipps R, et al. Soluble CD40 ligand accumulates in stored blood components, primes neutrophils through $\mathrm{CD} 40$, and is a potential cofactor in the development of transfusionrelated acute lung injury. Blood. 2006;108(7):2455-62. [PMID 16772606]

22. Chin-Yee I, Keeney M, Krueger L, Dietz G, Moses G. Supernate from stored red cells activates neutrophils. Transfus Med. 1998;8(1):49-56. [PMID 9569460]

23. Silliman CC, Voelkel NF, Allard JD, Elzi DJ, Tuder RM, Johnson JL, et al. Plasma and lipids from stored packed red blood cells cause acute lung injury in an animal model. J Clin Invest. 1998;101(7):1458-67. [PMID 9525989\}

24. Gajic O, Rana R, Mendez JL, Rickman OB, Lymp JF, Hubmayr RD, et al. Acute lung injury after blood transfusion in mechanically ventilated patients. Transfusion. 2004;44(10):1468-74. [PMID 15383020]

25. Huang $\mathrm{CH}$, Hu HC, Hsieh MJ, Huang $\mathrm{CT}$, Cho HY, Hsiao HF, et al. High-frequency oscillatory ventilation for rescue from refractory hypoxemia in a patient with transfusion-related acute lung injury. Respir Care. 2012;57(5):798-801. [PMID 22152999]

26. Wallis JP. Transfusion-related acute lung injury: presentation, epidemiology and treatment. Intensive Care Med. 2007;33 Suppl 1:S12-6. [PMID 17676434]

27. Litell JM, Gong MN, Talmor D, Gajic 0 . Acute lung injury: prevention may be the best medicine. Respir Care. 2011;56(10):1546-54. [PMID 22008396] 